\title{
Phase II Trial of Humanized Anti-Lewis $Y$ Monoclonal Antibody for Advanced Hormone Receptor-positive Breast Cancer That Progressed on Previous Endocrine Therapy
}

\section{Laura Testa ( $\sim$ lauratesta@gmail.com )}

Instituto do Câncer do Estado de São Paulo, Av. Dr. Arnaldo, 251, São Paulo

Max Mano

Instituto do Cancer do Estado de Sao Paulo

Roberto Jun Arai

Instituto do Cancer do Estado de Sao Paulo

Renata Colombo Bonadio

Instituto do Cancer do Estado de Sao Paulo https://orcid.org/0000-0001-5818-922X

\section{Sergio V. Serrano}

Hospital de Cancer de Barretos - Fundaçao Pio XII

Marina M. Costa Zorzetto

Hospital de Cancer de Barretos - Fundaçao Pio XII

\section{Susanne Crocamo}

Instituto Nacional do Cancer

\section{Oren Smaletz}

Recepta Biopharma and Hospital Israelita Albert Einstein

\section{Ruffo Freitas-Junior}

Federal University of Goias

Paulo M. Hoff

Instituto do Cancer do Estado de Sao Paulo

Research article

Keywords: Lewis Y antigen, breast cancer, tumoral expression of Lewis $Y$ antigen

Posted Date: November 19th, 2020

DOl: https://doi.org/10.21203/rs.3.rs-109611/v1

License: (c) (1) This work is licensed under a Creative Commons Attribution 4.0 International License. Read Full License 
Page $2 / 18$ 


\section{Abstract}

Purpose: Lewis $Y$ antigen is expressed in 44 to $90 \%$ of breast cancer. The expression of the antigen in carcinoma tissue is different from what occurs in normal tissue. The objective of the present study was to evaluate the clinical benefit of the humanized anti-Lewis Y monoclonal antibody, hu3S193, in advanced hormone receptor-positive and Lewis Y-positive breast cancer, after progression on endocrine therapy.

Methods: A single-arm phase II study was conducted in 7 centers. Patients with advanced hormone receptor-positive breast cancer who had failed previous first-line endocrine therapy were included. All patients were required to present tumoral expression of Lewis $Y$ antigen by immunohistochemistry. Treatment consisted of hu3S193 antibody at intravenous doses of $20 \mathrm{mg} / \mathrm{m} 2$ weekly given at 8-week cycles. The primary endpoint was clinical benefit rate.

Results: The study stopped accrual after an unplanned interim analysis since the hu3S193 antibody lacked sufficient activity to justify the study continuation. Twenty-two patients were enrolled; 21 were included in the efficacy analysis. The clinical benefit rate was $19 \%$, with four patients presenting stable disease confirmed after 24 weeks. One patient received the medication for more than 2 years, with prolonged stable disease. No partial or complete responses were observed. Median time to progression and overall survival were 5.4 and 37.5 months, respectively.

Conclusions: The humanized anti-Lewis Y monoclonal antibody, hu3S193, showed insufficient activity in this cohort. However, we cannot rule out the possibility of activity in a more strictly selected subgroup of patients with higher levels of Lewis Y tumoral expression.

Trial registration: Clinicaltrials.gov, NCT01370239. Registered 9 June 2011,

https://clinicaltrials.gov/ct2/show/NCT01370239

\section{Introduction}

Breast cancer (BC) is the most frequent cancer and the leading cause of cancer death among women ${ }^{1}$. The disease is divided into different subtypes with prognostic and therapeutic implications. Estrogen receptor expression occurs in $65 \%$ of invasive ductal carcinomas and in most cases of lobular carcinomas $^{2}$. For advanced hormone-receptor positive $\mathrm{BC}$, endocrine therapy $(\mathrm{ET})$ is the standard first-line treatment ${ }^{3-5}$. Recently, ET treatment has been improved by the incorporation of targeted therapies such as cyclin-dependent kinases (CDKs) inhibitors and mTOR inhibitors ${ }^{6-11}$. However, these treatment strategies are not widely available in public health systems yet ${ }^{12}$.

After the failure of first-line treatment, response to subsequent lines of ET or cytotoxic chemotherapy decreases dramatically. Treatment with second-line ET alone is associated with a median progressionfree survival of only 3 to 5 months, with response and clinical benefit rates in the range of $20-30 \%$ and 
$30-40 \%$, repectively ${ }^{13-17}$. This highlights the importance of incorporating the new treatment strategies in clinical practice as well as of studying novel targets.

Alterations of antigens related to blood groups are often found in association with neoplastic transformation ${ }^{18}$. Lewis $Y$ antigen is a member of a family of blood-related antigens. In adults, its expression is restricted to granulocytes and epithelial surfaces ${ }^{19}$. However, the antigen is expressed on the surface of $60-90 \%$ of the carcinomas ${ }^{20}$. In BC, expression of the Lewis $Y$ antigen occurs in $44-90 \%$ of cases, with variations occurring according to the methodology used 18,21,22. In addition, in BC, its expression correlates with a worse prognosis and a more advanced stage of the disease ${ }^{18,23}$.

Although expressed in both normal and neoplastic cells, the Lewis $Y$ antigen expression distribution differs between the two tissue types. Expression in normal epithelial tissue is restricted to the secretory borders of epithelial surfaces, making it less accessible to circulation. On the other hand, the expression of the antigen in carcinoma cells is very high in all surfaces, including luminal surfaces. This differentiated expression makes the Lewis $Y$ antigen an attractive target for treatment with monoclonal antibodies. This hypothesis is reinforced by preclinical studies that demonstrated the preferential localization of anti-Lewis $Y$ antibodies in tumor tissue, after its injection into the circulation of experimental animals ${ }^{24-26}$.

Considering these data, the murine monoclonal antibody 3S193 was developed to recognize the Lewis $Y$ antigen of the MCF-7 breast carcinoma cell line. Preclinical studies confirmed the specificity of the murine $3 S 193$ antibody to Lewis $Y$ antigen and its reactivity against cells expressing the antigen ${ }^{27}$. Therefore, a humanized IgG monoclonal antibody, the hu3S193, was developed ${ }^{20}$. Phase I trials demonstrated the safety of the hu3S193 antibody and preliminary evidence of activity in several tumor types, including $\mathrm{BC}^{28,29}$.

The objective of the present study was to evaluate the efficacy of the hu3S193 monoclonal antibody in patients with advanced hormone receptor-positive BC, after progression on previous ET.

\section{Patients And Methods}

\section{Patients}

The study included patients with metastatic or locally advanced BC not amenable to curative treatment, with tumoral expression of estrogen receptor, progesterone receptor, or both, by immunohistochemistry (IHC). Patients should have progressed after at least one line of ET, including adjuvant ET. Prior treatment with up to one line of chemotherapy for metastatic disease was acceptable.

All patients should have tumor expression of Lewis $\mathrm{Y}$ antigen documented by IHC. Tumor expression of the Lewis $Y$ antigen by IHC was performed by central analysis (LIM 14 of the Medical School of the 
University of São Paulo). The samples were considered positive when any reaction in the membrane of tumor cells was detected.

Other inclusion criteria were ECOG (Eastern Cooperative Oncology Group) performance status of 0 or 1 and preserved organic functions.

Exclusion criteria included the presence of HER2 overexpression (defined as IHC $3+$ or positive fluorescence in situ hybridization), life-threatening visceral metastatic disease (defined as extensive hepatic involvement, symptomatic pulmonary lymphangitic carcinomatosis, and cerebral or leptomeningeal metastases) and the need for systemic corticosteroids or immunosuppressive agents.

\section{Outcomes}

The primary endpoint was the clinical benefit rate of hu3S193 monoclonal antibody defined as complete response, partial response, or stable disease for at least 24 weeks.

Secondary endpoints were response rate, non-progression rate, overall survival, time to progression, and safety. The response rate was defined as the proportion of patients that presented complete response or partial response. The non-progression rate was defined as the proportion of patients who presented complete response, partial response or stable disease, regardless of the duration of the latter.

Overall survival was the time from the first study drug dose until death from any cause. Patients without this event were censored at the date of last follow-up. Time to progression was the time from first study drug dose until any clinical or radiological progression. Patients without the event were censored at the date of last follow-up or the date of death without progression.

\section{Study Design}

HumanaH trial was a phase II, single-arm, trial conducted in 7 Brazilian centers and coordinated by the Instituto do Câncer do Estado de São Paulo (ICESP) (NCT01370239).

Treatment consisted of hu3S193 antibody at intravenous infusions of $20 \mathrm{mg} / \mathrm{m} 2$ weekly. Each treatment cycle consisted of 8 weeks. Treatment was continued until clinical or radiological disease progression, unacceptable toxicity, withdrawal of consent, or decision of the investigator. Dose reductions were allowed according to protocol. In case of grades 3 or 4 non-allergic toxicities, treatment was delayed for up to 14 days until improvement to grades 1 or 2 and resumed with a $25 \%$ dose reduction. In case of no improvement to grades 1 or 2 within 14 days, treatment was discontinued.

\section{Study Supervision}

The Ethics Committee of each participating institution approved the study, which was conducted in accordance with the principles of the Declaration of Helsinki and the International Conference on Harmonisation Good Clinical Practice guidelines. Before any study procedure, the patients read and signed the Informed Consent Form. 
Recepta Biopharma (S. Paulo, Brazil) granted the use of hu3S193 antibodies. The study was sponsored by the Conselho Nacional de Desenvolvimento Científico e Tecnológico (CNPq), Grant N ${ }^{0}$ 52/2009 edital CNPq.

The authors were responsible for the design of the study, analysis of results, and writing the manuscript.

\section{Assessments}

Radiological response evaluations were performed every 8 weeks. Mandatory radiological images consisted of computed tomography or magnetic resonance imaging of the thorax, abdomen and pelvis. Brain imaging and bone scan were performed if clinically indicated. The radiological responses were evaluated according to Response Evaluation Criteria in Solid Tumors (RECIST), version 1.1.

Adverse events were monitored and their severity was scaled according to the National Cancer Institute Common Terminology Criteria for Adverse Events (CTCAE), version 4.03.

Human anti-human antibodies (HAHA) tests were performed periodically at baseline, at week 8 of each cycle, at the end of treatment, and if immune-mediated adverse events occurred. Samples were analyzed by ELISA (Enzyme linked Immunosorbent Assay). Treatment with hu3S193 antibody should be interrupted permanently if the tested patient was positive for HAHA.

\section{Statistical analysis}

Efficacy analyses were performed according to the intent-to-treat principle. In the safety analysis, all patients receiving at least one dose of the hu3S193 were included.

For sample size calculation, we considered historical clinical benefit rates obtained in three studies that included a similar profile of patients who were treated with endocrine therapy ${ }^{13,14,30}$. It was estimated a historical clinical benefit rate of $40 \%$ and a clinical benefit rate of hu3S193 of $60 \%$. With a two-sided alpha error of $5 \%$, a power of $85 \%$, and a withdrawal rate of $10 \%$, a total of 60 patients would be required.

Due to the observation that many patients soon discontinued the study because of disease progression, an unplanned interim analysis was done. The interim analysis considered a two-sided alpha error of $5 \%$, a beta error of $20 \%$, and clinical benefit rates of $40 \%$ historically and $60 \%$ with hu3S193. According to Simon's Two-Stage design, after testing 21 patients at a first stage, the trial should be terminated if 10 or fewer patients responded. If 11 or more patients responded, the trial should go on to the second stage to include a total of 60 patients.

Descriptive statistics were used to summarize patients' characteristics, radiological response, and adverse events. Continuous variables were presented as median and range, while categorical variables were presented as absolute and relative frequencies. Clinical benefit and non-progression rates were presented as proportion and $95 \%$ confidence interval $(\mathrm{Cl})$. Survival analyses were performed with the Kaplan-Meier method. Stata software, version 14 (StataCorp, Texas, USA), was used for statistical analyses. 


\section{Results}

\section{Patients characteristics}

From November 2013 to July 2015, 49 patients were screened for inclusion in the trial, of which 23 were initially considered eligible. One eligible patient was lost to follow-up before treatment began. Twenty-two patients received at least one dose of hu3S193 and were included in the safety analyses.

One of these 22 patients was incorrectly included in the trial since she had interstitial lung disease mistakenly considered as lung metastasis. This patient was not included in the efficacy analysis since she did not meet the study elegibility criteria. The remaining 21 patients were included in the efficacy analyses. The CONSORT diagram is presented in Fig. 1.

The median age was 54 years (range 39 to 79 years). All patients had estrogen receptor-positive and 17 patients $(77.3 \%)$ had progesterone receptor-positive BC. Sixteen patients $(72.7 \%)$ had received prior chemotherapy. The patients' characteristics are summarized in Table 1. 
Table 1

Patients characteristics

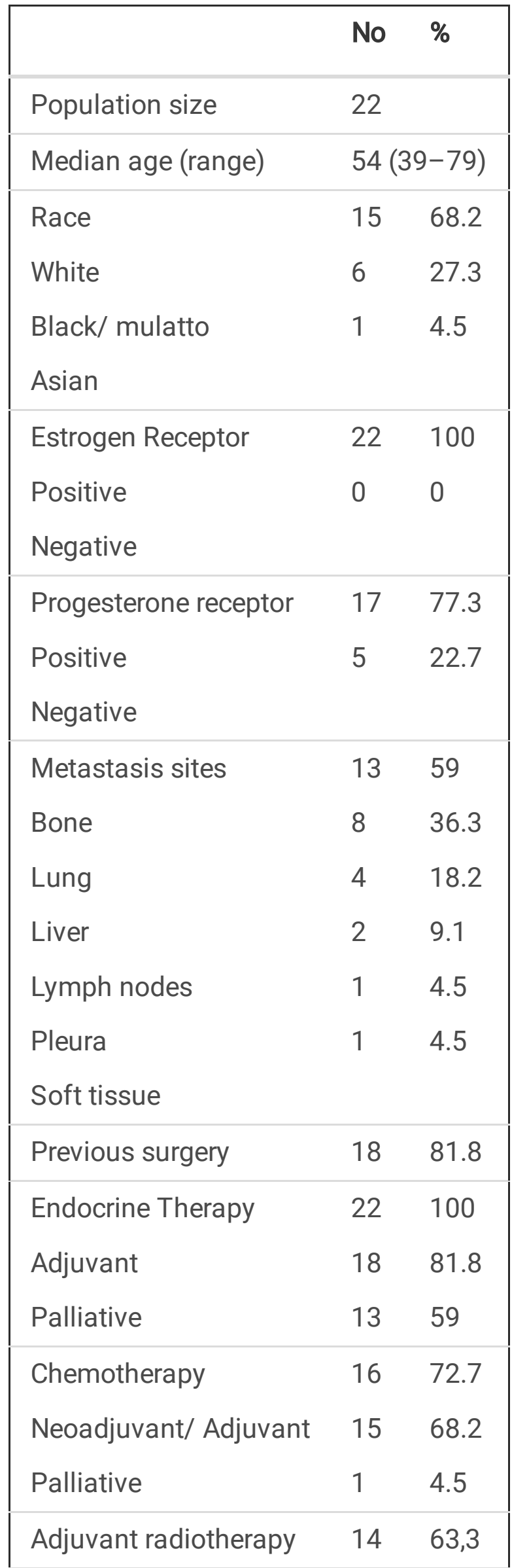




\begin{tabular}{|l|}
\hline \multicolumn{1}{|c|}{ No $\%$} \\
\hline Abbreviations: No, number. \\
\hline
\end{tabular}

\section{Efficacy}

The study was stopped after an unplanned interim analysis due to futility. The hu3S193 antibody did not show sufficient activity to justify the continuation of the study.

The median follow-up was 19 months at the time of study interruption. Four patients presented clinical benefit with hu3S193 antibody, corresponding to a clinical benefit rate of $19 \%(95 \% \mathrm{Cl}, 2.3 \%-35.8 \%)$. All patients presenting clinical benefit had stable disease for at least 24 weeks. One patient received the medication for more than 2 years, with stable disease. This patient presented a high expression of Lewis $\mathrm{Y}$ antigen (Fig. 2). No patient presented partial or complete responses. The non-progression rate was $57.1 \%(95 \% \mathrm{Cl}, 36 \%-78.3 \%)$. The radiological responses are summarized in Table 2. The median time to progression was 5.4 months ( $95 \% \mathrm{Cl}, 1.7-18.9$ months).

Table 2

Radiological response in the intention-to-treat population of patients with advanced hormone-receptor positive breast cancer treated with anti-Lewis Y monoclonal antibody (hu3S193)

\begin{tabular}{|lll|}
\hline & No of patients & $\%$ \\
\hline Complete response & $(\mathbf{N}=\mathbf{2 1})$ & \\
\hline Partial response & 0 & 0 \\
\hline Stable disease (SD) & 0 & $57.1 \%$ \\
SD as best radiological response & 12 & $19 \%$ \\
SD confirmed after 24 weeks & 4 & $38.1 \%$ \\
\hline Disease progression (as best radiological response) & & $4.8 \%$ \\
\hline Not evaluable* & 8 & \\
\hline $\begin{array}{l}\text { Abbreviations: No, number; SD, stable disease. } \\
\text { * One patient presented with clinical worsening in the first weeks of treatment and had no imaging } \\
\text { evaluation after treatment. }\end{array}$ & 1 & \\
\hline
\end{tabular}

Overall survival data was updated after a median follow-up of 31.1 months. Fourteen patients deceased during this period. The median overall survival was 37.5 months $(95 \% \mathrm{Cl}, 15.5$ months - not reached). Time to progression and overall survival curves are presented in Fig. 3.

\section{Adverse events}


Two patients had serious adverse events potentially related to study treatment. One had dyspnea and the other, vomiting, dyspnea, and infections (two episodes of pneumonia and one of urinary tract infection). Both patients recovered properly.

All 22 patients who received at least one dose of treatment had non-serious adverse events potentially related to study treatment. The most frequent adverse events were headache $(N=11)$, cough $(N=10)$, nausea $(N=7)$, vomiting $(N=7)$, and musculoskeletal pain $(N=6)$. Major events (grades 3 and 4) were uncommon. Adverse events by grade are presented in Table 3. 
Table 3

Adverse events by grade in patients with advanced hormone-receptor positive breast cancer treated with anti-Lewis Y monoclonal antibody (hu3S193).

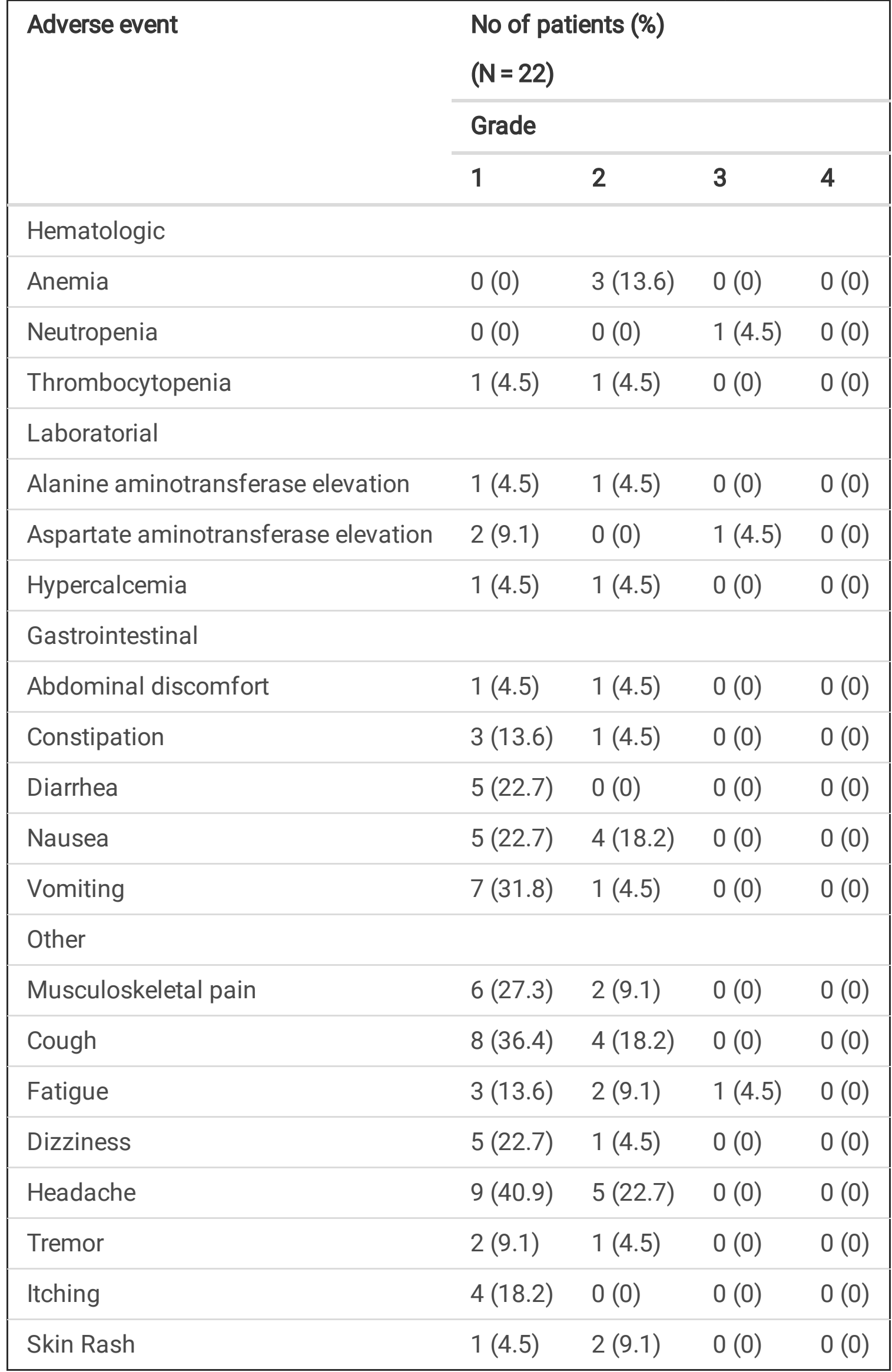


There was no death related to study treatment.

\section{Discussion}

In the present phase II study, patients with advanced hormone receptor-positive BC who had progressed after previous ET were treated with the humanized antibody targeting the Lewis $Y$ antigen hu3S193. This agent did not show sufficient activity to justify the continuation of the study, which was closed after an unplanned interim analysis due to futility. Of the 21 patients included in the study's efficacy population, only 4 had stable disease confimed after 24 weeks and none had partial or complete responses (clinical benefit rate of $19 \%)$.

Similar results were reported in another phase II study that evaluated the efficacy of hu3S193 in patients with advanced, platinum-resistant, ovarian, tubal or primary peritoneal cancer ${ }^{31}$. This study was also prematurely interrupted due to low drug activity, with a clinical benefit rate of $23 \%$ (stable disease confirmed at 24 weeks) and no documented partial or complete responses.

However, one patient presented a prolonged clinical benefit with the treatment, maintaining stable disease after two years of hu3S193 antibody treatment. This patient had elevated levels of Lewis Y tumor expression. Thus, a possibility exists that a more strictly selected patient population (characterized by elevated Lewis $Y$ tumoral expression) could still benefit from the treatment.

In terms of safety, treatment with hu3S193 antibody proved to be safe and well tolerated. The adverse events considered related to the study treatment were mostly low-grade and manageable, consisting mainly of headaches, cough, musculoskeletal pain, nausea, and vomiting. No serious adverse events mediated by the immune system were seen.

It is important to highlight that, after the present study was ongoing, significant changes occurred in the treatment of hormone receptor-positive $\mathrm{BC}$ due to the introduction of cyclin-dependent kinase inhibitors (CDK) as mentioned previously. Currently, the benefit of the addition of these drugs to ET has been demonstrated in both first-line and second-line settings ${ }^{6-11}$. Due to robust gains in progression-free survival, three drugs of this class (palbociclib, ribociclib, and abemaciclib) has changed the treatment algorithm for advanced/metastatic estrogen receptor-positive breast cancer and have been incorporated into clinical practice in many countries $6,32,33$.

In conclusion, the anti-Lewis $Y$ antibody hu3S193 showed insufficient efficacy in patients with advanced hormone receptor-positive and Lewis $\mathrm{Y}$ antigen-positive $\mathrm{BC}$ who had progressed on previous ET. Activity in patients with higher levels of Lewis $Y$ antigen tumoral expression cannot be rulled out. No relevant toxicity with hu3s193 was observed.

\section{Declarations}


Ethical Approval and Consent to participate: Ethics approval was obtained from Ethics Committee of each participating institution. All participants gave informed consent to participate before taking part of the study.

Consent for publication: not applicable.

Availability of supporting data: All data from the study are included in this article, or available upon reasonable request from the corresponding author.

Disclosures: RCB has received financial support for educational programs from AstraZeneca and financial support for attending symposia from Roche, outside the submitted work. All other authors have no disclosure/ conflict of interest.

Funding: This work received financial support provided by Brazilian National Council of Scientific and Technology Development (CNPq), edital 52/2009.

Authors' contributions: Concept and design: LT, MM, RJA, OS, and PMH; patient accrual: LT, MM, SVS, MMCZ, SC, RFJ; data acquisition and analysis: LT, MM, RJA, RCB, PMH; manuscript writing: LT, MM, RJA, $\mathrm{RCB}$, and RFJ. All authors read and approved the final manuscript.

Acknowledgements: We acknowledge all patients for participation in the study. We also thank Juliana Guimarães, Debora Pupo da Fonseca Silveira, Elaine de Santana Longo, Indrani Majunder, Vivian Madrigal, and Mariana Nigro Fiuza for invaluable clinical operations support.

\section{References}

1. Bray F, Ferlay J, Soerjomataram I, Siegel RL, Torre LA, Jemal A. Global cancer statistics 2018 : GLOBOCAN estimates of incidence and mortality worldwide for 36 cancers in 185 countries. CA Cancer J Clin 2018; 68: 394-424.

2. Luveta J, Parks RM, Heery DM, Cheung KL, Johnston SJ. Invasive Lobular Breast Cancer as a Distinct Disease: Implications for Therapeutic Strategy. Oncol Ther. 2020 Jun; 8(1):1-11.

3. Lei W, Li H, Song G, Zhang R, Ran R, Yan Y, Di L, Jiang H. Efficacy and Safety of Fulvestrant 500mg in Hormone-receptor Positive Human Epidermal Receptor 2 Negative Advanced Breast Cancer: A Realworld Study in China. J Cancer. 2020 Sep 23;11(22):6612-6622

4. Robertson JFR, Cheung KL, Noguchi S, Shao Z, Degboe A, Lichfield J, Thirlwell J, Fazal M, Ellis MJ. Health-related quality of life from the FALCON phase III randomised trial of fulvestrant $500 \mathrm{mg}$ versus anastrozole for hormone receptor-positive advanced breast cancer. Eur J Cancer. 2018 May;94:206215.

5. Katz A, Saad ED, Porter P, et al: Primary systemic chemotherapy of invasive lobular carcinoma of the breast. Lancet Oncol 8:55-62, 2007 
6. Schneeweiss A, Ettl J, Lüftner D, Beckmann MW, Belleville E, Fasching PA, et al. Initial experience with CDK4/6 inhibitor-based therapies compared to antihormone monotherapies in routine clinical use in patients with hormone receptor positive, HER2 negative breast cancer - Data from the PRAEGNANT research network for the first 2 years of drug availability in Germany. Breast. 2020 Aug 29;54:88-95.

7. Cristofanilli M, Turner NC, Bondarenko I, et al: Fulvestrant plus palbociclib versus fulvestrant plus placebo for treatment of hormone-receptor-positive, HER2-negative metastatic breast cancer that progressed on previous endocrine therapy (PALOMA-3): final analysis of the multicentre, doubleblind, phase 3 randomised controlled trial. Lancet Oncol 17:425-439, 2016

8. Hortobagyi GN, Stemmer SM, Burris HA, et al: Ribociclib as First-Line Therapy for HR-Positive, Advanced Breast Cancer. N Engl J Med 375:1738-1748, 2016

9. Sledge GW, Toi M, Neven P, et al: MONARCH 2: Abemaciclib in Combination With Fulvestrant in Women With HR+/HER2- Advanced Breast Cancer Who Had Progressed While Receiving Endocrine Therapy. J Clin Oncol 35:2875-2884, 2017

10. Goetz MP, Toi M, Campone M, et al: MONARCH 3: Abemaciclib As Initial Therapy for Advanced Breast Cancer. J Clin Oncol 35:3638-3646, 2017

11. Baselga J, Campone M, Piccart M, et al: Everolimus in postmenopausal hormone-receptor-positive advanced breast cancer. N Engl J Med 366:520-9, 2012

12. Soares LR, Freitas-Junior R, Curado MP, Paulinelli RR, Martins E, Oliveira JC. Low Overall Survival in Women With De Novo Metastatic Breast Cancer: Does This Reflect Tumor Biology or a Lack of Access to Health Care? JCO Glob Oncol. 2020 Apr;6:679-687

13. Howell A, Robertson JF, Quaresma Albano J, et al: Fulvestrant, formerly ICI 182,780 , is as effective as anastrozole in postmenopausal women with advanced breast cancer progressing after prior endocrine treatment. J Clin Oncol 20:3396-403, 2002

14. Osborne CK, Pippen J, Jones SE, et al: Double-blind, randomized trial comparing the efficacy and tolerability of fulvestrant versus anastrozole in postmenopausal women with advanced breast cancer progressing on prior endocrine therapy: results of a North American trial. J Clin Oncol 20:3386-95, 2002

15. Iwamoto T, Fujisawa T, Shien T, Araki K, Sakamaki K, Sangai T, et al. The efficacy of sequential second-line endocrine therapies (ETs) in postmenopausal estrogen receptor-positive and HER2negative metastatic breast cancer patients with lower sensitivity to initial ETs. Breast Cancer. 2020 Sep;27(5):973-981

16. Reinert T, Barrios $\mathrm{CH}$. Overall survival and progression-free survival with endocrine therapy for hormone receptor-positive, HER2-negative advanced breast cancer: review. Ther Adv Med Oncol. 2017 Nov;9(11):693-709

17. Kaufmann M, Bajetta E, Dirix LY, et al: Exemestane is superior to megestrol acetate after tamoxifen failure in postmenopausal women with advanced breast cancer: results of a phase III randomized double-blind trial. The Exemestane Study Group. J Clin Oncol 18:1399-411, 2000 
18. Madjd Z, Parsons T, Watson NF, et al: High expression of Lewis $y / b$ antigens is associated with decreased survival in lymph node negative breast carcinomas. Breast Cancer Res 7:R780-7, 2005

19. Dettke M, Pálfi G, Loibner H: Activation-dependent expression of the blood group-related lewis $Y$ antigen on peripheral blood granulocytes. J Leukoc Biol 68:511-4, 2000

20. Scott AM, Geleick D, Rubira M, et al: Construction, production, and characterization of humanized anti-Lewis $Y$ monoclonal antibody $3 S 193$ for targeted immunotherapy of solid tumors. Cancer Res 60:3254-61, 2000

21. Hellström I, Garrigues HJ, Garrigues U, et al: Highly tumor-reactive, internalizing, mouse monoclonal antibodies to Le(y)-related cell surface antigens. Cancer Res 50:2183-90, 1990

22. Saleh MN, Sugarman S, Murray J, et al: Phase I trial of the anti-Lewis $Y$ drug immunoconjugate BR96-doxorubicin in patients with lewis Y-expressing epithelial tumors. J Clin Oncol 18:2282-92, 2000

23. Steplewska-Mazur K, Gabriel A, Zajecki W, et al: Breast cancer progression and expression of blood group-related tumor-associated antigens. Hybridoma 19:129-33, 2000

24. Clarke $\mathrm{K}$, Lee FT, Brechbiel MW, et al: In vivo biodistribution of a humanized anti-Lewis $Y$ monoclonal antibody (hu3S193) in MCF-7 xenografted BALB/c nude mice. Cancer Res 60:4804-11, 2000

25. Lövqvist A, Humm JL, Sheikh A, et al: PET imaging of (86)Y-labeled anti-Lewis Y monoclonal antibodies in a nude mouse model: comparison between (86) $\mathrm{Y}$ and (111)In radiolabels. J Nucl Med 42:1281-7, 2001

26. Pai-Scherf LH, Carrasquillo JA, Paik C, et al: Imaging and phase I study of $111 \mathrm{In}$ - and $90 \mathrm{Y}$-labeled anti-LewisY monoclonal antibody B3. Clin Cancer Res 6:1720-30, 2000

27. Kitamura K, Stockert E, Garin-Chesa P, et al: Specificity analysis of blood group Lewis-y (Le(y)) antibodies generatedagainst synthetic and natural Le(y) determinants. Proc Natl Acad Sci U S A 91:12957-61, 1994

28. Scott AM, Tebbutt N, Lee FT, et al: A phase I biodistribution and pharmacokinetic trial of humanized monoclonal antibody Hu3s193 in patients with advanced epithelial cancers that express the Lewis-Y antigen. Clin Cancer Res 13:3286-92, 2007

29. Krug LM, Milton DT, Jungbluth AA, et al: Targeting Lewis $Y(\operatorname{Le}(y))$ in small cell lung cancer with a humanized monoclonal antibody, hu3S193: a pilot trial testing two dose levels. J Thorac Oncol 2:947-52, 2007

30. Chia S, Gradishar W, Mauriac L, et al: Double-blind, randomized placebo controlled trial of fulvestrant compared with exemestane after prior nonsteroidal aromatase inhibitor therapy in postmenopausal women with hormone receptor-positive, advanced breast cancer: results from EFECT. J Clin Oncol 26:1664-70, 2008

31. Smaletz O, Diz MD, do Carmo CC, et al: A phase II trial with anti-Lewis-Y monoclonal antibody (hu3S193) for the treatment of platinum resistant/refractory ovarian, fallopian tube and primary peritoneal carcinoma. Gynecol Oncol 138:272-7, 2015 
32. Giordano SH, Elias AD, Gradishar. WJ NCCN Guidelines Updates: Breast Cancer. J Natl Compr Canc Netw. 2018 May;16(5S):605-610

33. Gao JJ, Cheng J, Bloomquist E, Sanchez J, Wedam SB, Singh H, et al. CDK4/6 inhibitor treatment for patients with hormone receptor-positive, HER2-negative, advanced or metastatic breast cancer: a US Food and Drug Administration pooled analysis. Lancet Oncol. 2020 Feb;21(2):250-260

\section{Figures}

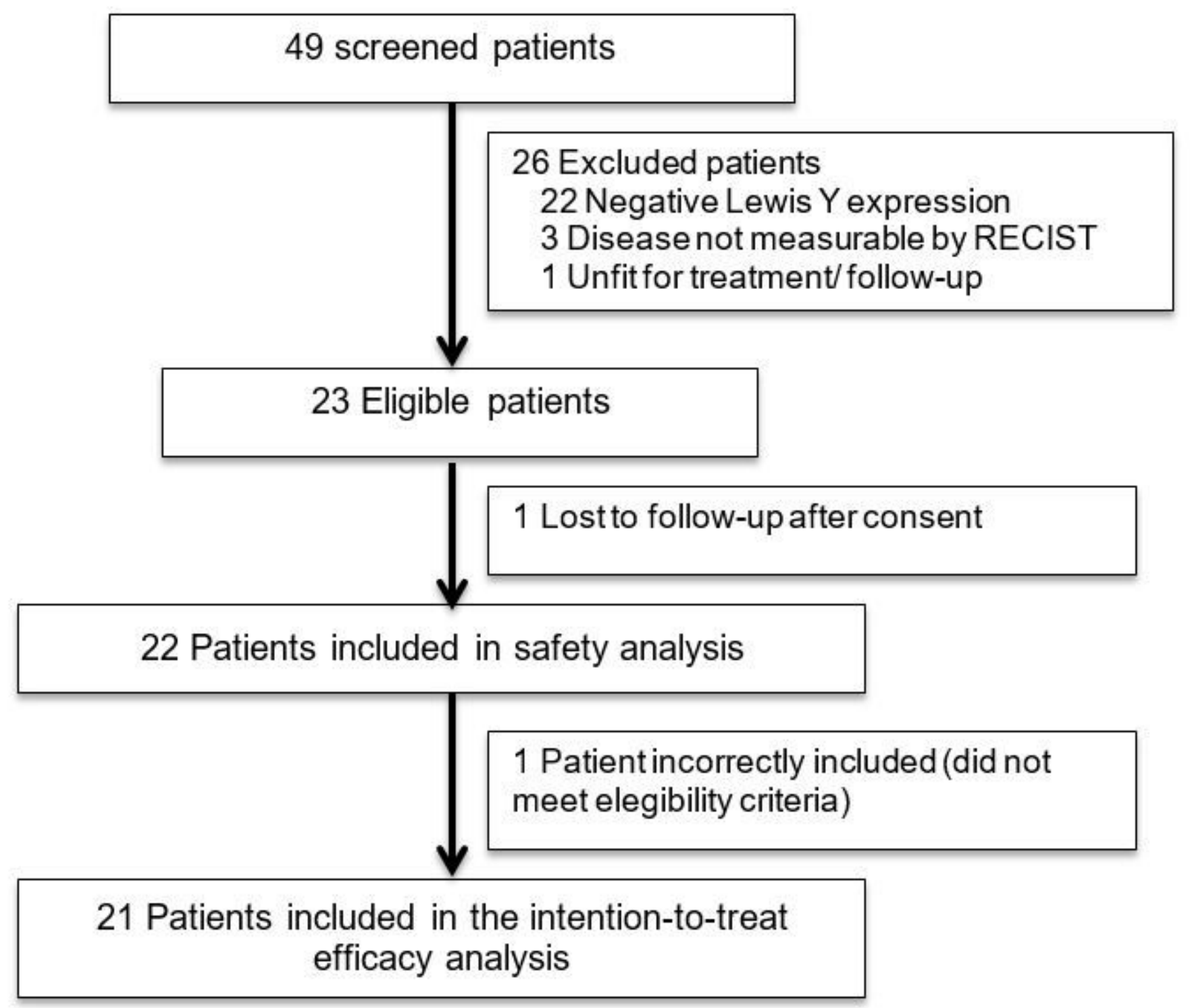

Figure 1

CONSORT diagram. RECIST, Response Evaluation Criteria in Solid Tumors. 

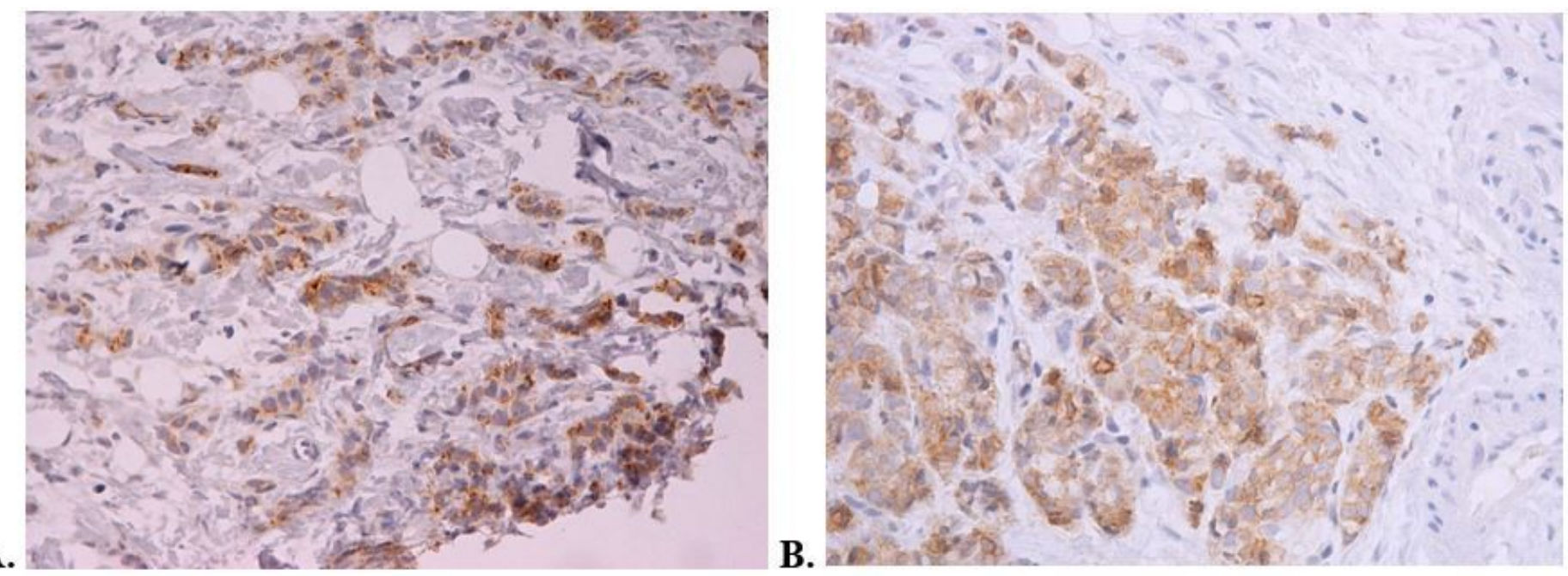

\section{Figure 2}

Lewis $Y$ antigen expression by immunohistochemistry of the patient that received hu3S193 with stable disease for more than 2 years. A. Predominant Membrane Lewis Y staining X 200. B. Membrane and Cytoplasm Lewis Y staining X 200. 

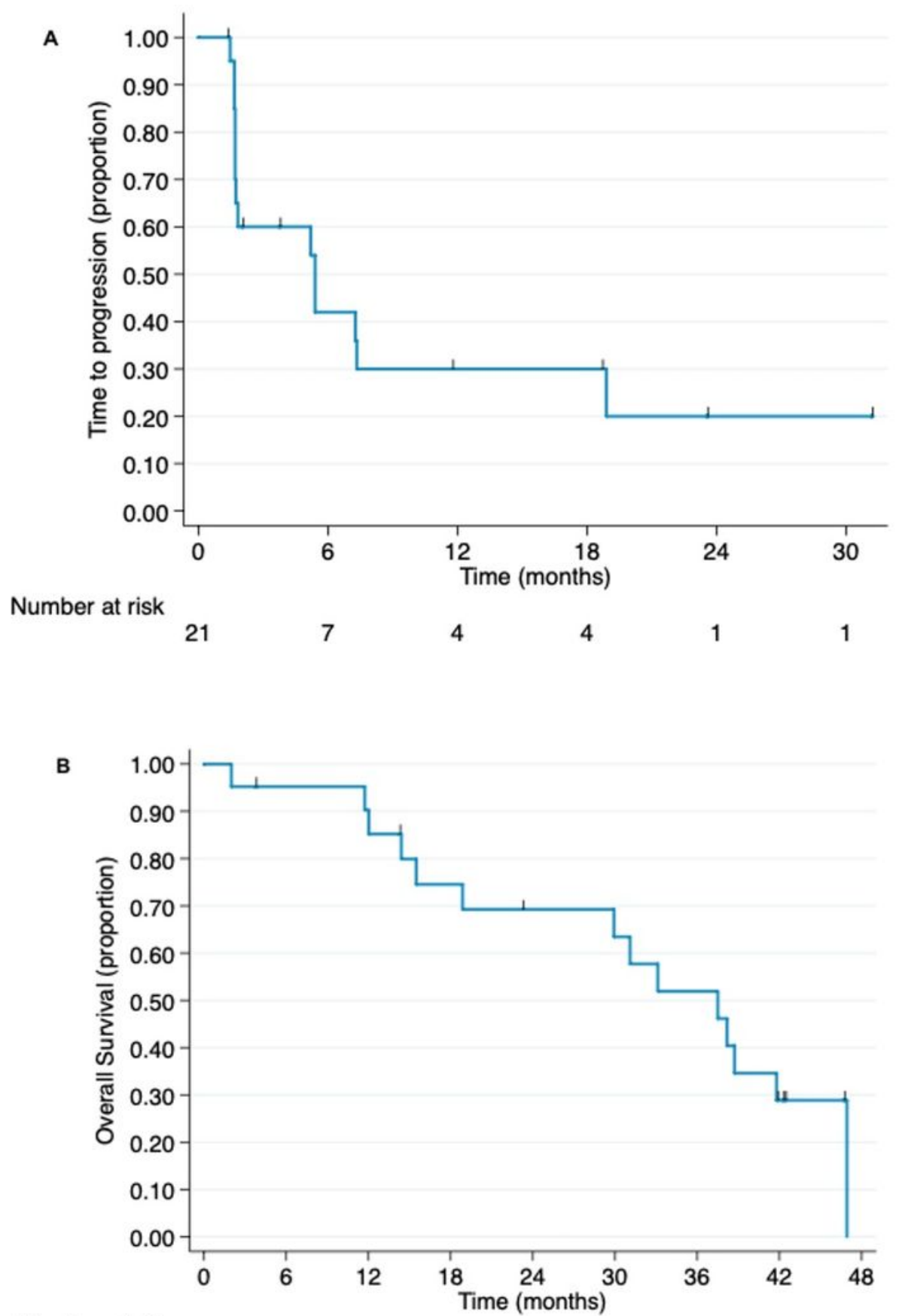

Number at risk

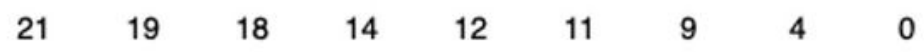

\section{Figure 3}

Kaplan-Meier curves for (A) Time to progression and (B) Overall Survival for patients with advanced hormone-receptor positive breast cancer treated with anti-Lewis Y monoclonal antibody (hu3S193) (intention-to-treat population). 